\title{
Ultraslow Group Velocity and Enhanced Nonlinear Optical Effects in a Coherently Driven Hot Atomic Gas
}

\section{Citation}

Kash, Michael M., Vladimir A. Sautenkov, Alexander S. Zibrov, L. Hollberg, George R. Welch, Mikhail D. Lukin, Yuri Rostovtsev, Edward S. Fry, and Marlan O. Scully. 1999. "Ultraslow Group Velocity and Enhanced Nonlinear Optical Effects in a Coherently Driven Hot Atomic Gas." Physical Review Letters 82 (26): 5229-32. https://doi.org/10.1103/physrevlett.82.5229.

\section{Permanent link}

http://nrs.harvard.edu/urn-3:HUL.InstRepos:41467463

\section{Terms of Use}

This article was downloaded from Harvard University's DASH repository, and is made available under the terms and conditions applicable to Other Posted Material, as set forth at http:// nrs.harvard.edu/urn-3:HUL.InstRepos:dash.current.terms-of-use\#LAA

\section{Share Your Story}

The Harvard community has made this article openly available.

Please share how this access benefits you. Submit a story.

\section{Accessibility}


Group Delay-NLO

\title{
Ultra-Slow Light and Enhanced Nonlinear Optical Effects in a Coherently Driven Hot Atomic Gas.
}

\author{
Michael M. Kash, ${ }^{1,5}$ Vladimir A. Sautenkov, ${ }^{1}$ Alexander S. Zibrov, ${ }^{1,3}$ L. Hollberg, ${ }^{3}$ \\ George R. Welch, ${ }^{1}$ Mikhail D. Lukin,${ }^{4}$ Yuri Rostovtsev, ${ }^{1}$ Edward S. Fry, ${ }^{1,2}$ \\ Marlan O. Scully ${ }^{1,2}$ \\ ${ }^{1}$ Department of Physics, Texas A $3 M$ University, College Station, Texas 77843-4242 \\ 2 Max-Planck-Institut für Quantenoptik, D-85748 Garching, Germany \\ ${ }^{3}$ National Institute for Standards and Technology, Boulder, Colorado 80303 \\ ${ }^{4}$ ITAMP, Harvard-Smithsonian Center for Astrophysics, Cambridge, Massachusetts 02138 \\ ${ }^{5}$ Department of Physics, Lake Forest College, Lake Forest, Illinois 60045
}

(February 1, 2008)

\begin{abstract}
We report the observation of small group velocities of order 90 meters per second, and large group delays of greater than $0.26 \mathrm{~ms}$, in an optically dense hot rubidium gas $(\approx 360 \mathrm{~K})$. Media of this kind yield strong nonlinear interactions between very weak optical fields, and very sharp spectral features. The result is in agreement with previous studies on nonlinear spectroscopy of dense coherent media.
\end{abstract}

PACS numbers 42.50.-p, 42.55.-f, 42.50.Gy

Typeset using REVTEX 
A phase coherent ensemble of atoms ("phaseonium,") represents a truly novel state of matter. A dramatic example of such a quantum coherence effect is provided by the recent report of extremely slow group velocity $(17 \mathrm{~m} / \mathrm{s})$ for a pulse of light in a Bose condensate of ultra-cold sodium atoms [1]. Previous direct and indirect measurements of increasingly low group velocities in coherently prepared media have ranged from c/165 [2], and c/3000 [3] to about $\mathrm{c} / 10^{6}$ 四. In this report we show that by a proper choice of experimental parameters such as atomic density and optical intensity, very large group delay (slow group velocity) of light can be observed in a cell of hot $(360 \mathrm{~K}){ }^{87} \mathrm{Rb}$ atoms. This is in agreement with our previous studies of spectroscopy and magnetometry in dense coherent media.

Furthermore, we demonstrate here that this relatively easily created medium also displays very strong nonlinear coupling between very weak optical fields [5]. Specifically, with such a thermal ensemble of rubidium atoms, we observe (1) group delay $\left(T_{g}\right)$ of $0.26 \mathrm{~ms}$ for propagation through our $2.5 \mathrm{~cm}$ long, optically thick, electromagnetically-induced transparent (EIT) medium, and (2) extremely efficient nonlinear interactions. These two aspects of phaseonium are closely related: $T_{g}$ will be shown to be the figure of merit for various linear and nonlinear optical processes using EIT (see e.g. Eq. (77) and Ref. [5]). Specific manifestations of these unusual properties of dense coherent media include new regimes of high precision spectroscopy and nonlinear interactions of the very weak light fields [6.] with greatly alleviated phase matching requirements [8]

We observed large group delay on the $\mathrm{D} 1$ resonance line $(\lambda=795 \mathrm{~nm})$ of ${ }^{87} \mathrm{Rb}$ (nuclear spin $\mathrm{I}=3 / 2$ ). The cell contained isotopically pure ${ }^{87} \mathrm{Rb}$ and 30 Torr of Ne buffer gas. Under this condition, with a $2 \mathrm{~mm}$ laser beam diameter, the ground-state coherence relaxation rate $\gamma_{b c} /(2 \pi)$ is reduced below $1 \mathrm{kHz}$. The measured time delay as a function of the power of the drive input to the cell is shown in Fig. 1. The drive laser was tuned to the $5^{2} \mathrm{~S}_{1 / 2}(\mathrm{~F}=2) \rightarrow 5^{2} \mathrm{P}_{1 / 2}(\mathrm{~F}=2)$ transition; a co-propagating probe laser was tuned to the $5^{2} \mathrm{~S}_{1 / 2}(\mathrm{~F}=1) \rightarrow 5^{2} \mathrm{P}_{1 / 2}(\mathrm{~F}=2)$ transition (see Fig. 20a). Both of these lasers were external cavity diode lasers. They were phase-locked with a frequency offset near the ground-state hyperfine splitting of $6.8 \mathrm{GHz}$, which was fixed by a tunable microwave frequency synthesizer. The 
probe laser power was $5 \%$ of the drive laser power, and was amplitude modulated by approximately $50 \%$ with a sine wave at a frequency that was varied in the range of $0.1-10 \mathrm{kHz}$.

Figure 11 also shows the inferred average group velocity for each measured delay time. As the lasers propagate down the length of the cell, the drive laser power is attenuated. Since the group velocity decreases with drive laser power (see Eq. (5) and Fig. 31), we see that the instantaneous velocity is lower toward the output end of the cell than near the input. Hence, we report the average velocity in the cell.

The group delay in passing through the cell was measured by observing the time retardation of the amplitude modulation upon passing through the cell. The attenuation and time delay was measured for a range of modulation frequencies, allowing us to model the propagation for a wide range of pulses. The time delay was independent of the modulation frequency up to the linewidth of the EIT resonance. Systematic effects resulting in unwanted phase shifts of the amplitude modulation of the light were investigated by several approaches: cooling the cell so that very little Rb vapor was present, tuning far from resonance, removing the cell, and checking the electronics for spurious phase shifts. A schematic of the experimental setup is shown in Fig. $2 \mathrm{~b}$.

In the experiment, both the drive beam and the probe beam are transmitted through the cell, and because of nonlinear optical processes additional frequencies are generated by the medium as discussed below. To isolate the amplitude of the transmitted probe, we split off part of the drive before the cell and shift its frequency down by a small amount $(50 \mathrm{MHz})$ as indicated in Fig $2 \mathrm{~b}$. This shifted beam bypasses the cell and is combined on the detector along with the transmitted drive and probe and any generated fields. Because the amplitude of the shifted field is constant, this signal is proportional to the transmitted probe without any contribution from the transmitted drive field.

The low group velocity arises from the large dispersion of the coherent medium. For a light field with a slowly changing complex amplitude, we write $E(z, t)=\mathcal{E}(z, t) \exp (i k z-$ $i \nu t)$, and $P(z, t)=\mathcal{P}(z, t) \exp (i k z-i \nu t)$, where $\mathcal{E}(z, t)$ and $\mathcal{P}(z, t)$ are the slowly varying envelopes of the electric field and atomic polarization. The carrier wave has wavenumber 
$k$ and frequency $\nu$. The Fourier components of the field and the polarization are related by $P(z, \nu)=\epsilon_{0} \chi(\nu) E(z, \nu)$, where $\chi(\nu)$ is the susceptibility of the medium. Substituting these relations into the wave equation and neglecting all derivatives greater than the first, we obtain the equation of motion for the envelope:

$$
\left(\frac{\partial}{\partial z}+\frac{1}{v_{g}} \frac{\partial}{\partial t}\right) \mathcal{E}(z, t)=i \frac{k}{2} \chi(\nu) \mathcal{E}(z, t) .
$$

Traditionally, the susceptibility is divided into real and imaginary parts: $\chi=\chi^{\prime}+i \chi^{\prime \prime}$, and vanishingly small $\chi^{\prime}$ and $\chi^{\prime \prime}$ at resonance are the signature of EIT. The group velocity in the medium is given by $v_{g}=c /\left[1+(\nu / 2)\left(d \chi^{\prime} / d \nu\right)\right]$, where the derivative is evaluated at the carrier frequency.

For a medium displaying EIT, $\chi\left(\nu_{p}\right)$ is given by [9]

$$
\chi\left(\nu_{p}\right)=\int_{-\infty}^{\infty} \frac{i \eta \gamma_{r} \Gamma_{b c}}{\Gamma_{b c}\left[\gamma+i\left(\Delta_{p}+k_{p} v\right)\right]+\Omega^{2}} f(v) d v
$$

In this expression $\nu_{p}$ is the probe laser frequency, $\eta=\left(3 \lambda^{3} N\right) /\left(8 \pi^{2}\right)$ where $\lambda$ is the probe wavelength and $N$ is the atomic density, $\Gamma_{b c}=\gamma_{b c}+i\left[\delta+\left(k_{p}-k_{d}\right) v\right]$ where $\gamma_{r}$ is the radiative decay rate of level $a$ to level $b, \gamma_{b c}$ is the coherence decay rate of the two lower levels, (governed here by the time-of-flight through the laser beams), $\gamma$ is the total homogeneous half-width of the drive and probe transitions (including radiative decay and collisions), $\Delta_{p}=\omega_{a b}-\nu_{p}$ and $\Delta_{d}=\omega_{a c}-\nu_{d}$ are the one-photon detunings of the probe and drive lasers, and $\delta=\Delta_{p}-\Delta_{d}$ is the two-photon detuning, $\Omega$ is the Rabi frequency of the drive transition, $k_{p}$ and $k_{d}$ are wave numbers of the probe and driving fields respectively. One can obtain a simple analytic expression corresponding to Eq. (2) by approximating the thermal distribution $f(v)$ by a lorentzian, $f(v)=(k v / \pi) /\left[\left(\Delta \omega_{D}\right)^{2}+(k v)^{2}\right]$, where $\Delta \omega_{D}$ is the Doppler half-width of the thermal distribution and $v$ is the projection of the atomic velocity along the laser beams. The result is

$$
\chi\left(\nu_{p}\right)=\eta \gamma_{r} \frac{i \gamma_{b c}-\delta}{\left(\gamma+\Delta \omega_{D}+i \Delta_{p}\right)\left(\gamma_{b c}+i \delta\right)+\Omega^{2}}
$$

where we have taken $k=k_{p}=k_{d}$. 
Equation (3) leads to propagation with absorption coefficient $\alpha=(k / 2) \chi^{\prime \prime}\left(\nu_{p}\right)$ and group velocity $v_{g}=c /\left(1+n_{g}\right)$. We obtain

$$
\begin{aligned}
\alpha & =\frac{3}{8 \pi} N \lambda^{2} \frac{\gamma_{r} \gamma_{b c}}{\gamma_{b c}\left(\gamma+\Delta \omega_{D}\right)+\Omega^{2}}, \\
n_{g} & =\frac{3}{8 \pi} N \lambda^{2} \frac{\gamma_{r} \Omega^{2} c}{\left[\gamma_{b c}\left(\gamma+\Delta \omega_{D}\right)+\Omega^{2}\right]^{2}} .
\end{aligned}
$$

After propagation through a dense coherent ensemble of length $L$ the intensity of the pulse is attenuated by $\exp (-2 \alpha L)$, whereas its envelope is delayed compared to free space propagation by $T_{g}=n_{g} L / c$.

To relate the present results to earlier studies of EIT-based spectroscopy and prior group delay measurements, we note that the group delay is essentially the reciprocal of the socalled "dispersive width" associated with the EIT resonance $\Delta \omega_{\text {dis }}=\pi /\left(2 T_{g}\right)$. This width was defined in Ref. [4] as the detuning from the line center at which the phase of the probe laser shifts by $\pi / 2$. The significance of this quantity is that it determines the ultimate resolution of interferometric measurements using EIT. When the group delay is large, the dispersive width is correspondingly small. This is the basis for high-precision spectroscopy in dense coherent media.

Clearly, an essential difference between hot and cold atom experiments concerns Doppler broadening. Equation (5) shows that for our experimental regime where $\Omega^{2} \gg \gamma_{b c}\left(\gamma+\Delta \omega_{D}\right)$ the effect of Doppler averaging is not important. The point is that in many current experiments (EIT, LWI, high resolution dense medium spectroscopy, and ultra-slow group velocities) the results are two-photon Doppler free for copropagating drive and probe fields. That is, as shown in Eq. (2), when $k_{p} \approx k_{d}$, only the single photon denominator (the squarebracketed expression in Eq. (21) depends on atomic velocity. This has a negligible effect near two photon resonance, provided that the Rabi-frequency of the driving field is sufficiently large. This analysis, our experimental demonstration, and numerical calculations, allow us to conclude that for strong driving fields, the effects of Doppler averaging are not of central importance to the group velocity. The results of numerical calculations are shown in Fig. 3 . For our current experiments, in which $\gamma_{b c} /(2 \pi) \approx 10^{3} \mathrm{~Hz}$ and $\left(\gamma+\Delta \omega_{D}\right) /(2 \pi) \approx 4 \times 10^{8} \mathrm{~Hz}$, 
drive Rabi frequencies $\Omega /(2 \pi) \gg 10^{6} \mathrm{~Hz}$ are required in order to get a measurable signal through the cell. We note from Fig. 3 that for this range of intensity, the lorentzian approximation holds, and also that $\partial \chi^{\prime} / \partial \nu$ is nearly the same for hot and cold gases. Furthermore, curve (d) in Fig. 司 shows that for our current experimental conditions, a reduction of $\gamma_{b c}$ allows the possibility of reaching much lower group velocities, near $10 \mathrm{~m} / \mathrm{s}$. Such a reduction in $\gamma_{b c}$ is quite possible by increasing our laser beam diameter as shown in Ref. [10].

On the other hand, the cold atom technology does hold promise for a truly Doppler free payoff; e.g., nonlinear optical processes involving "sideways" coupling [1,5] in which the drive and probe lasers are perpendicular. This is not possible in a hot gas. Likewise, EIT experiments in cold gases might be a very interesting tool for studying the properties of and even manipulating the Bose condensate.

We next turn to nonlinear interactions such as wave mixing involving pulses or $\mathrm{cw}$ fields in a phase coherent media. In the present system, nonlinear wave mixing phenomenon can be induced by the off-resonant coupling of states $a$ and $b$ in Fig. 2a by the driving field, or by applying a second driving field $\Omega_{2}$. As discussed previously [⿴囗十, [1] two important situations should be distinguished. They correspond to driving fields with Rabi frequencies $\Omega_{1}$ and $\Omega_{2}$ that propagate in the same or opposite directions, respectively. In the case of counter-propagating fields, oscillation occurs [12]. In the case of co-propagating fields at high optical power and $\mathrm{Rb}$ vapor density, coherent Raman scattering leads to efficient

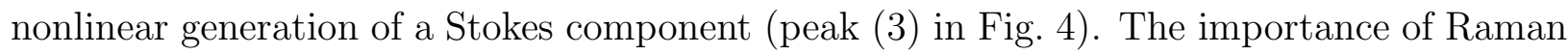
nonlinearities in such resonant wave mixing phenomena has been discussed previously [13].

Evidence of this generation for the case of co-propagating fields has been observed for cw fields [4]. There, the off-resonant coupling of the drive field to the $a \leftrightarrow b$ transition led to generation of a new (Stokes) field. The new field is generated at $\nu_{d}-\omega_{c b}$ (see Fig. 2aa). In Ref. [4] this new field was observed indirectly by observing the simultaneous beat between the drive, probe, and new fields at $\omega_{c b}$.

By using the method described above for isolating the strength of the transmitted probe field, we can now isolate the strength of the generated new field. Figure 4 shows the relative 
strengths of the probe and new field. It is striking to note that, under conditions of a large group delay, the output power in these two fields (1 and 3) is nearly the same. Furthermore, the properties of this field may be studied with the current experimental arrangement with a time-varying probe, and will be discussed in detail elsewhere.

We emphasize the connection between ultra-slow light propagation and large nonlinearities. As in Fig. 4 , consider the case of co-linear propagation of the slowly varying anti-Stokes (i.e. probe) and Stokes (i.e. new) fields, coupled by coherent Raman scattering. We assume that the cw driving field is near resonance with the $c \leftrightarrow a$ transition. In the vicinity of two-photon resonance the probe field $\mathcal{E}_{p}$ and the new Stokes field $\mathcal{E}_{n}$ evaluated at the exit from the cell of length $L$ is given by [1]:

$$
\begin{aligned}
\mathcal{E}_{p} & =\mathcal{E}_{p}(0) \cosh \left(\xi T_{g}\right) \\
\mathcal{E}_{n}^{*} & =i \mathcal{E}_{p}(0) \sinh \left(\xi T_{g}\right)
\end{aligned}
$$

where $\xi=\Omega^{2} / \omega_{c b}$ and for simplicity we have ignored loss and chosen the detuning $\delta$ such that phase-matching is satisfied (i.e. $\left.\delta=\left(k_{p}+k_{n}-2 k_{d}\right) c / n_{g}\right)$. This indicates that the medium with a sufficiently long-lived ground state coherence $\gamma_{b c}$, and sufficiently large density-length product (to insure large group delay, and consequently large nonlinear gain) is required to achieve efficient nonlinear generation. We emphasize that these requirements are typical for any efficient nonlinear interactions involving phase coherent media. Dramatic examples are large Kerr nonlinearities [14], single photon switching [6], and quantum control and correlations of weak laser beams [0]].

In conclusion we have demonstrated ultra-large group delay $T_{g} \approx 0.26 \mathrm{~ms}$ for light traversing a cell containing an ensemble of hot phase coherent atoms for which the transit time through an empty cell is a fraction of a nanosecond. Such a phaseonium gas has ultralarge nonlinear optical properties yielding nonlinear coupling between very weak fields. It is safe to predict that such phase coherent materials will be of both fundamental (e.g. probing the Bose condensate) and applied (e.g. compression of information by many orders of magnitude) interest. 
This work was supported by the Office of Naval Research, the National Science Foundation, the Robert A. Welch Foundation, and the U. S. Air Force. We thank Steve Harris for his enthusiasm concerning the present studies resulting in this paper, and G. Agarwal, C. Bednar, S. Harris, L. Hau, H. Lee, and A. Matsko for stimulating and helpful discussions. 


\section{REFERENCES}

[1] L. V. Hau, S. E. Harris, Z. Dutton, and C. H. Behroozi, Nature 397, 594 (1999).

[2] A. Kasapi, M. Jain, G. Y. Yin, and S. E. Harris, Phys. Rev. Lett. 74, 2447 (1995).

[3] O. Schmidt, R. Wynands, Z. Hussein and D. Meschede, Phys. Rev. A 53, R27 (1996).

[4] M. D. Lukin, M. Fleischhauer, A. S. Zibrov, H. G. Robinson, V. L. Velichansky, L. Hollberg, and M. O. Scully, Phys. Rev. Lett. 79, 2959 (1997).

[5] S. E. Harris and L. V. Hau, Nonlinear Optics at a Low Light Level, (submitted).

[6] S. E. Harris, Y. Yamamoto, Phys. Rev. Lett. 81, 3611 (1998).

[7] A. Imamoğlu, H. Schmidt, G. Woods, and M. Deutsch, Phys. Rev. Lett. 79, 1467 (1997);

M. Dunstan, S. Rebic, S. Tan, S. Parkins, M. Collett, and D. Walls, in Proceedings: Quantum Communication, Computing, and Measurement 2. (ed. by P. Kumar, G. M. D'Ariano, and O. Hirota); M. D. Lukin, A. Matsko, M. Fleischhauer, M. O. Scully, Phys. Rev. Lett. 82, 1847 (1999).

[8] M. Jain, G. Y. Yin, J. E. Field, S. E. Harris, Opt. Lett., 18, 998 (1993).

[9] See for example chapter 7 of M. O. Scully and M. S. Zubairy, "Quantum Optics", Cambrige University Press 1997.

[10] S. Brandt, A. Nagel, R. Wynands, and D. Meschede, Phys. Rev. A 56, R1063 (1997).

[11] M. D. Lukin, P. R. Hemmer, M. Lffler, and M. O. Scully, Phys. Rev. Lett. 81, 2675 (1998).

[12] "Parametric Self-Oscillation via Resonantly Enhanced Multiwave Mixing", A. S. Zibrov, M. D. Lukin, and M. O. Scully, submitted, (1999).

[13] D. J. Gauthier, M. S. Malcuit, and R. W. Boyd, Phys. Rev. Lett. 61, 1827 (1988).

[14] H. Schmidt, A. Imamoğlu, Opt. Lett., 21, 1936 (1996). 


\section{FIGURES}

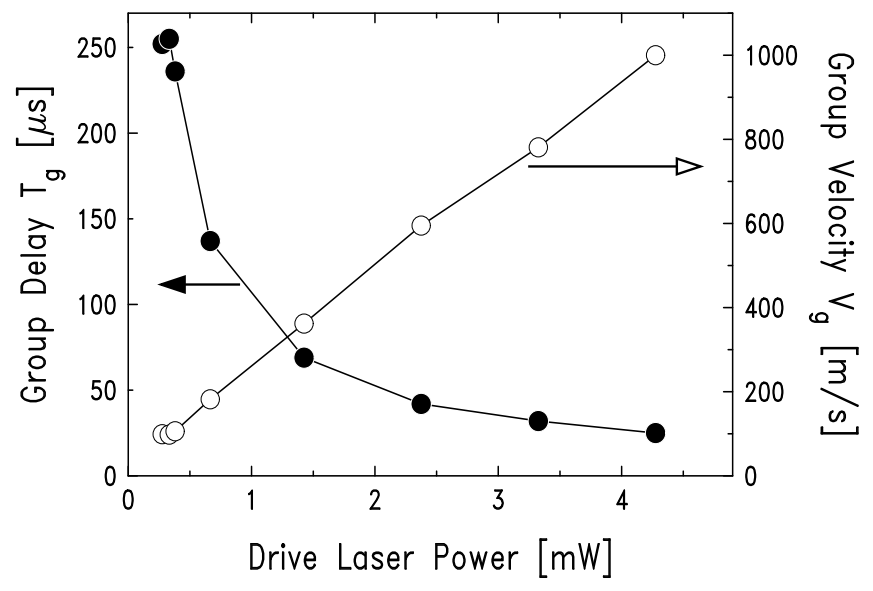

FIG. 1. Observed group delay (solid circles) and average group velocity (open circles) as a function of the drive laser power. The density of ${ }^{87} \mathrm{Rb}$ was $2 \times 10^{12} \mathrm{~cm}^{-3}$ and the laser beam diameter was $2 \mathrm{~mm}$. For this transition, $\Omega /(2 \pi)=1 \times 10^{6} \sqrt{I}$ where $I$ is in $\mathrm{mW} / \mathrm{cm}^{2}$.

(a)

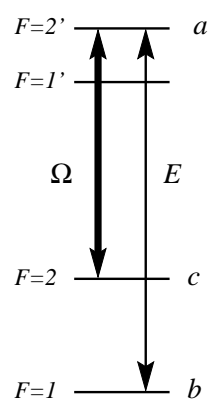

(b)

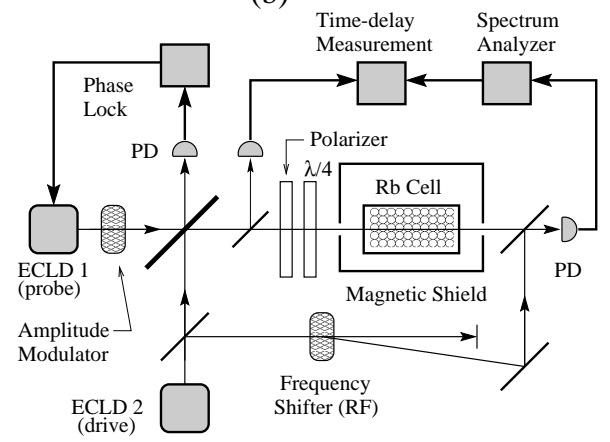

FIG. 2. (a) Level scheme for group delay measurement. (b) Schematic of the experiment. The PDs represent high speed photo-detectors. Amplitude Modulator and Frequency Shifter are acousto-optic modulators. 


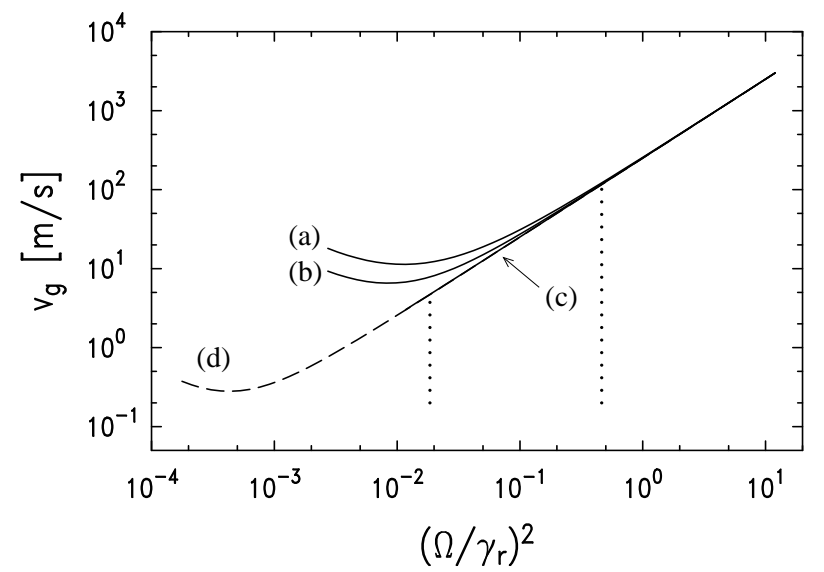

FIG. 3. Calculated group velocity versus drive laser power for $\mathrm{Rb}$ with density $N=2 \times 10^{12} \mathrm{~cm}^{-3}$. Curves (a), (b), and (c) are calculated with lorentzian, gaussian, and no Doppler averaging respectively. A ground state relaxation rate $\gamma_{b c} /(2 \pi)=1000 \mathrm{~Hz}$, is assumed for all three. A collisionally broadened homogeneous half-width $\gamma /(2 \pi)=150 \mathrm{MHz}$ and Doppler half-width $\Delta \omega_{D} /(2 \pi)=270 \mathrm{MHz}$ is used for curves (a) and (b), and the radiative half-width $\gamma /(2 \pi)=\gamma_{r} /(2 \pi)=3 \mathrm{MHz}$ is used for curve (c). For drive intensities below the right vertical dotted line, the medium is strongly opaque (absorption $>90 \%$ ). A key point is that for transparent media, the effect of Doppler averaging on $v_{g}$ is small. The dashed curve (d) is for the hot case as in (b), but with $\gamma_{b c} /(2 \pi)=40 \mathrm{~Hz}$, which is experimentally possible. For such a lower coherence decay, the onset of strong absorption should occur at lower intensity, indicated by the left vertical dotted line, allowing for much lower $v_{g}$.

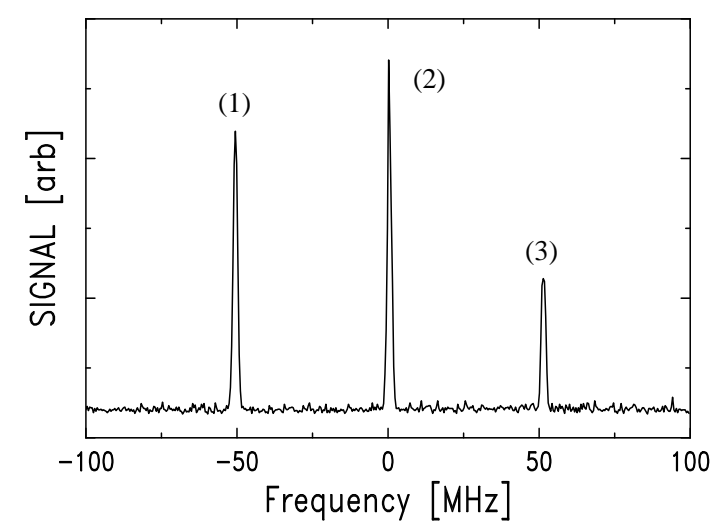


FIG. 4. RF spectrum for frequencies in the vicinity of the ground-state hyperfine splitting showin build-up of a "new"-field from the vacuum, plotted on a linear scale. Peak (1) is the beat between the probe and shifted fields, peak (2) is the beat between the drive, probe, and new fields, and peak (3) is the beat between the new and shifted fields. Note that the amplitude of the the new field is comparable to the probe field indicating extremely efficient nonlinear generation. The drive power was $4.3 \mathrm{~mW}$. 